\title{
Role of dispersion in pulse emission from a sliding-frequency fiber laser
}

\author{
M. Romagnoli and S. Wabnitz \\ Fondazione Ugo Bordoni, via B. Castiglione 59, 00142 Roma, Italy \\ P. Franco and M. Midrio \\ Dipartimento di Elettronica e Informatica, Università di Padova, via G. Gradenigo 6/A, 35131 Padova, Italy \\ L. Bossalini and F. Fontana \\ Pirelli Cavi s.p.a., viale Sarca 202, 20146 Milano, Italy \\ Received October 20, 1994 \\ We present an experimental and theoretical investigation of the role of group-velocity dispersion in the \\ generation of picosecond pulses from a sliding-frequency fiber loop laser.
}

\section{INTRODUCTION}

The technique of generation of short optical pulses by means of a sliding-frequency (or frequency-shifted feedback) laser cavity ${ }^{1}$ was recently successfully applied to neodymium ${ }^{2}$ and erbium ${ }^{3-5}$ fiber laser systems. A schematic view of the various elements in a slidingfrequency fiber loop laser is shown in Fig. 1. Continuous-frequency sliding is achieved by an acoustooptic shifter like the one that is depicted in Fig. 2. In a fiber laser cavity with anomalous group-velocity dispersion (GVD), under certain conditions the propagation of the circulating pulses in a sliding-frequency fiber laser (SFFL) may be well described in terms of a perturbed nonlinear Schrödinger (NLS) equation. ${ }^{6}$ In fact, it was pointed out by Kodama et al. ${ }^{6}$ that in this case the pulse formation mechanism in a SFFL is closely related to the sliding-filter method that has been proposed and demonstrated by Mollenauer et al. ${ }^{7,8}$ for the stabilization of long-distance soliton transmission systems. Pulse stability in the laser configuration is due to the combination of a continuous-frequency shifter and a filter. After a few transits the continuous-frequency shifter removes low-intensity noise and continuous-wave radiation from the filter bandpass and hence from the cavity. But a soliton survives in the cavity because it remains stably trapped by the filter at a given frequency. The dynamics of the noise-induced start-up of frequency-shifted feedback fiber lasers was numerically studied by Sabert and Brinkmeyer. ${ }^{2,4}$ In the NLS limit discussed above, one may apply soliton perturbation theory to obtain a simple analytical expression for the steady-state pulse duration from the SFFL. ${ }^{3,6}$ Based on this analysis, one postulates that the laser pulse's time width is uniquely fixed by the frequency-shifting rate and the filter bandwidth. In other words, the duration of the output pulse is independent, for example, of the magnitude of the cavity dispersion and the cavity length.
The preliminary experimental results in Refs. 3 and 5 appear to agree quite well with this hypothesis.

Still to be investigated is the role of the average cavity GVD on the generation and stability of pulses from a SFFL in cases in which the operating wavelength is close to zero or takes positive values. Based on previous research on pulse formation in passively mode-locked laser systems, ${ }^{9-12}$ in this paper we study both theoretically and experimentally the effects of the sign and the magnitude of the cavity GVD on the pulse formation process in a SFFL. In particular, in agreement with the results of Refs. 2 and 4, we show here that stable pulses may be supported in a SFFL as a result of the interplay among bandwidth-limited amplification, frequency shifting, self-phase modulation, and fast saturable absorption even with zero or positive GVD. Moreover, by exploiting the compression of the chirped pulses that are generated in the SFFL with a positive cavity GVD by means of an anomalous GVD output fiber lead, we obtain the emission of near-transform-limited $400-f$ pulses at a repetition rate that is fixed by a the round-trip group delay in the loop.

\section{EXACT SOLUTIONS}

The averaged propagation equation for the field envelope $u$ with mean frequency $\omega_{0}$ in the cavity reads, in dimensionless units, as

$$
\begin{array}{r}
i \frac{\partial u}{\partial Z}-\frac{D}{2} \frac{\partial^{2} u}{\partial T^{2}}-i \beta \frac{\partial^{2} u}{\partial T^{2}}-i \delta u+(1+i \gamma)|u|^{2} u \\
-i \sigma|u|^{4} u+\alpha T u=0
\end{array}
$$

where $D=\beta_{2} /\left|\beta_{r}\right|$ is the average cavity GVD $\beta_{2}$ divided by the absolute value of a reference value of GVD, say, $\beta_{r}$. In addition, $\delta=g-l$ is the difference between gain and loss in the loop, $\beta$ is the average gain dispersion, $\gamma$ and $\sigma$ are the nonlinear gain and its saturation, 


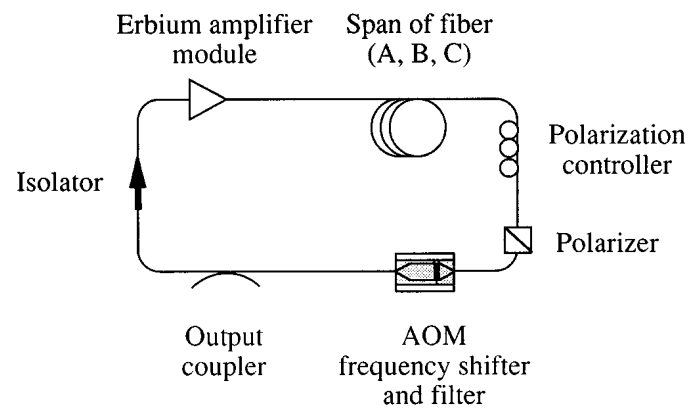

Fig. 1. Schematic of the laser. Three different fibers (A, B, C) were used to control the average GVD of the fiber loop: A, $L_{A}=110 \mathrm{~m}, \beta_{2}=-20 \mathrm{ps}^{2} / \mathrm{km} ; \mathrm{B}, L_{B}=58 \mathrm{~m}, \beta_{2}=0$ at $1550 \mathrm{~nm}$; and $\mathrm{C}, L_{C}=40 \mathrm{~m}, \beta_{2}=+83 \mathrm{ps}^{2} / \mathrm{km}$. AOM, acousto-optical modulator.

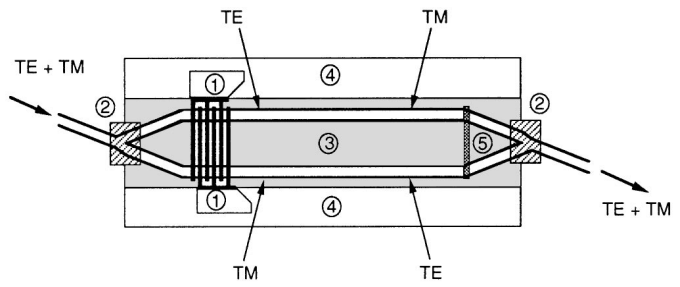

Fig. 2. Schematic of the acousto-optic modulator and filter (AOMF): (1) unidirectional transducer, (2) polarization beam splitter, (3)acoustic waveguide, (4)Ti-indiffused region, (5)acoustic absorber.

respectively (these terms represent the effect of a fast saturable absorption mechanism in the cavity ${ }^{9}$ ), and $\alpha$ is proportional to the frequency-sliding rate. Moreover, $T=\left(t-z / v_{g}\right) / t_{0}$ ( $t_{0}$ is an arbitrary reference time unit) and $Z=z / z_{0}\left(z_{0}=t_{0}^{2} /\left|\beta_{r}\right|\right.$ is the dispersion length). We denote the group velocity $v_{g}$. In Eq. (1) we may take into account the slow saturation of the active fiber medium by simply writing $g=g_{0} /\left(1+P / P_{s}\right)$, where $P$ is the average power in the cavity and $P_{s}$ is the saturation power. ${ }^{2,9,13}$ A detailed discussion of the stabilizing effect of slow gain saturation on the propagation pulses in fiber lasers was presented in Ref. 13. Here we are interested mainly in investigating the pulse stabilization that originates from a continuous-frequency sliding and/or a fast equivalent saturable-absorption mechanism; therefore in what follows we neglect analysis of the role of slow gain saturation.

In real units, the frequency-shifting rate $\alpha$ and the filter dispersion coefficient $\beta$ read as

$$
\alpha=\frac{\Delta f t_{0}{ }^{3}}{z_{l} \beta_{r}}, \quad \beta=\frac{2}{\Delta \Omega^{2} z_{l} \beta_{r}},
$$

where $\Delta f$ and $\Delta \Omega$ are the acousto-optic modulator angular frequency shift and the filter bandwidth $(2 \pi \mathrm{THz})$, respectively. Moreover, $t_{0}$ is expressed in picoseconds, $z_{l}$ is the loop length (here this is expressed in kilometers, and $\beta_{r}$ is given in square picoseconds per kilometer.

As we shall see, the main role of nonlinear gain saturation and of frequency sliding is in the stabilization of the pulses that circulate through the cavity. In fact, it has been shown that the essential ingredients for the formation of time-localized pulses in a physical system that is described by a generalized Ginzburg-Landau equation of the form of Eq. (1) are nonlinearity and bandwidthlimited amplification, irrespective of the sign of GVD. ${ }^{14,15}$ Neglecting at first for simplicity higher-order nonlinear gain saturation and frequency shifting, that is, with $\sigma=$ $\alpha=0$, one obtains an exact solution for Eq. (1) in the form $^{11,14}$

$$
u=A[\operatorname{sech}(T / \rho)]^{1-i \nu} \exp (i \Gamma Z)
$$

where

$$
\begin{aligned}
\nu & =-a \pm \sqrt{a^{2}+2}, \\
a & =\frac{3(\gamma \beta+D / 2)}{\beta-D \gamma / 2}, \\
\rho^{2} & =\left(\beta \nu^{2}-\beta+\nu D\right) / \delta, \\
A^{2} & =\left(D \nu^{2} / 2-D-3 \nu \beta\right) / \rho^{2}, \\
\Gamma & =\left(D \nu^{2} / 2-D / 2-2 \beta \nu\right) / \rho^{2} .
\end{aligned}
$$

Figures 3-8 illustrate the dependence of the pulse width $\rho$, the spectral bandwidth $\xi\left(=\sqrt{1+\nu^{2}} / \rho\right)$, and the amplitude $A$ of the solitary-wave solution [Eqs. (3) and (4)] on the dispersion coefficient $D$. In Figs. 3-5 the linear excess gain $\delta>0$, whereas in Figs. 6-8 $\delta<0$. In Figs. $3-5$ we set $\beta=3 \delta=0.15$ and we varied the nonlinear gain (or saturable loss) coefficient from $\gamma=0$ (dashed curves) to $\gamma=-0.035$ (solid curves) and $\gamma=-0.07$ (dotted-dashed curves). As can be seen from Fig. 3, the presence of a finite nonlinear gain reduces the pulse width $\rho$. This decrease in the pulse duration is more effective whenever the absolute value of $D$ grows larger. Note that without nonlinear gain the pulse width is almost constant (and close to 1) in the anomalous dispersion region (i.e., with $D<0$ ), whereas the pulse duration grows linearly with $D$ in the normal dispersion case. Figure 4 illustrates the dependence of the spectral width of the pulses as in Fig. 3 on the dispersion parameter $D$. As can be seen, in the anomalous GVD region the pulse remains close to a transform-limited NLS equation soliton, whereas with normal GVD the pulse's spectral width rapidly increases, although the nonlinear gain introduces some bandwidth reduction. Figure 5 shows the dependence on $D$ of the pulse amplitude $A$. The amplitude is nearly constant in the normal GVD regime, whereas $A$ rapidly diverges for negative GVD whenever the nonlinear gain grows larger (and correspondingly the pulse width is compressed; see Fig. 3).

On the other hand, Fig. 6 was obtained with negative excess gain $\delta$. Here $\beta=0.15, \delta=-0.02$, and $\gamma=-0.2$ (solid curve), $\gamma=-0.4$ (dashed curve), and $\gamma=-0.8$ (dotted-dashed curve). As can be seen, for $\gamma$ less than approximately 0.3 there is a range of dispersion values around $D=0$ where there is no localized solution of Eq. (1) of the type of Eqs. (3) and (4). Moreover, progressively larger absolute values of the nonlinear gain coefficient lead to longer pulse durations. In contrast with the case $\delta>0$, the pulse width also increases linearly in the anomalous dispersion regime as the absolute value of $D$ grows larger. Figure 7 displays the pulse spectral widths $\xi$ that are associated with the curves in Fig. 6: As can be seen, even for different values of $\gamma$ the behavior of the temporal and spectral widths is similar. Finally, 


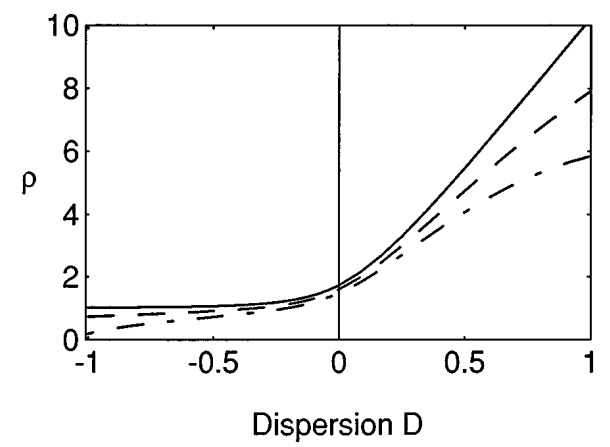

Fig. 3. Theoretical dependence of pulse width on the dispersion coefficient $D$ for $\beta=3 \delta=0.15$ and $\gamma=0$ (solid curve), $\gamma=-0.035$ (dashed curve), and $\gamma=-0.07$ (dotted-dashed curve).

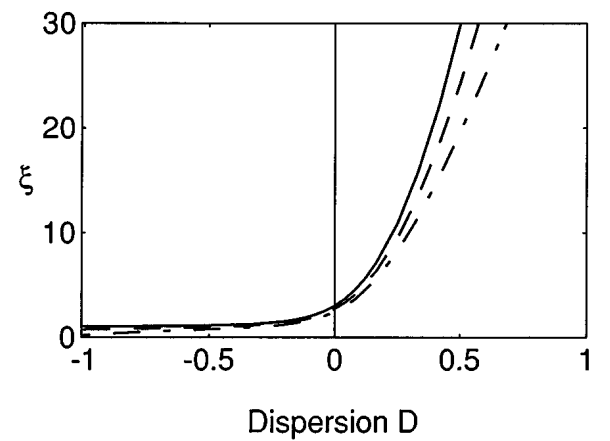

Fig. 4. Pulse spectral width $\xi=\sqrt{1+\nu^{2}} / \rho$ versus cavity dispersion $D$ for the same parameters as in Fig. 3.

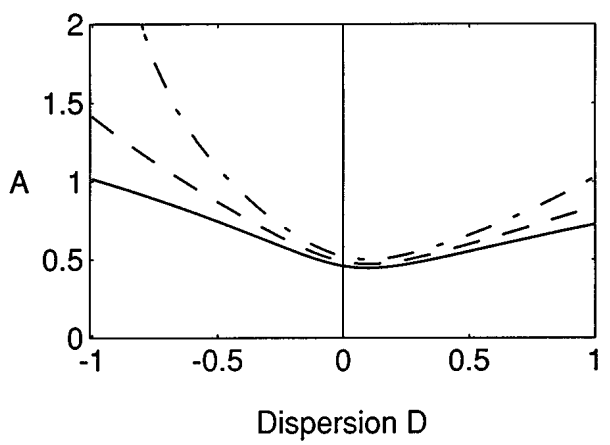

Fig. 5. Pulse amplitude $A$ versus dispersion $D$ for the parameters of Fig. 3.

the amplitude $A$ variation curves in Fig. 8 show that the vanishing pulse widths in Fig. 6 correspond to the limit of infinitely intense pulses, which is indicative of the collapse mechanism that is present in the solutions of the Ginzburg-Landau equation (1). Clearly, in a real laser system pulse collapse is prevented by nonlinear gain saturation terms like that in Eq. (1).

In the normal dispersion regime the pulses [Eqs. (3) and (4)] are strongly chirped; that is, their phase rapidly rotates along the pulse profile. For example, the solid curve in Fig. 9 shows the variation of the instantaneous frequency $C \doteq \mathrm{d}[\arg (u)] / \mathrm{d} T=\mathrm{d}(\nu \ln \{\cosh (T / \rho)]\} / \mathrm{d} T$ across the pulse amplitude (dashed curve) for $\delta=-0.02$, $\beta=0.16, \gamma=-0.2$, and $D=1$. As can be seen, the frequency change or chirp is almost linear in the central region of the pulse. As will be experimentally demonstrated later in this paper, Fig. 9 shows that pulse com- pression could be achieved by propagation of this pulse through an anomalous dispersive fiber.

A key issue in the physical meaning of the solution [Eqs. (3) and (4)] is its stability on propagation. In par-

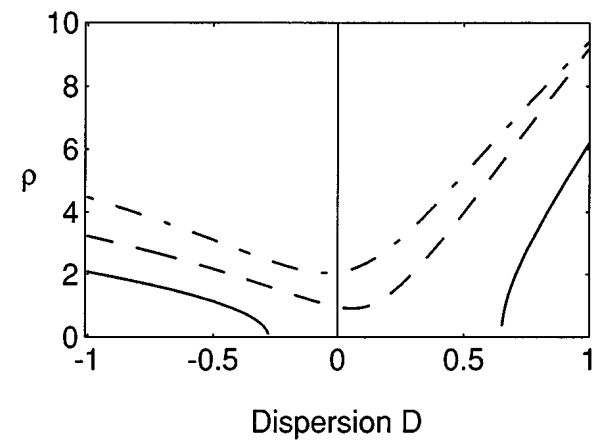

Fig. 6. Same as in Fig. 3 for $\beta=0.16, \delta=-0.02$, and $\gamma=-0.2$ (solid curve), $\gamma=-0.4$ (dashed curve), and $\gamma=-0.8$ (dotted-dashed curve).

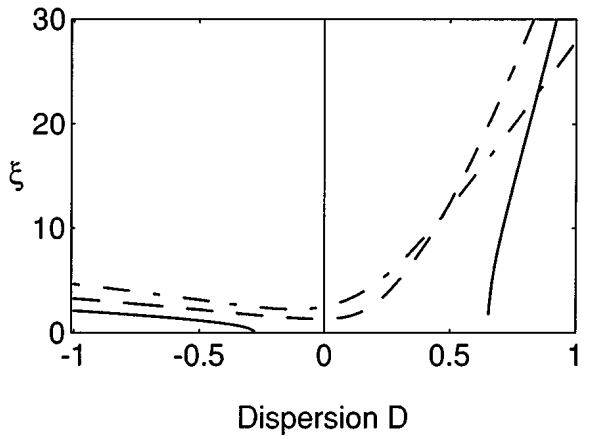

Fig. 7. Same as in Fig. 4 for the parameters of Fig. 6 .

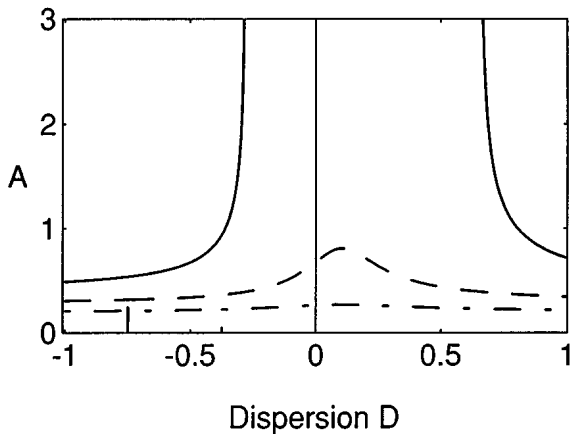

Fig. 8. Same as in Fig. 5 for the parameters of Fig. 6 .

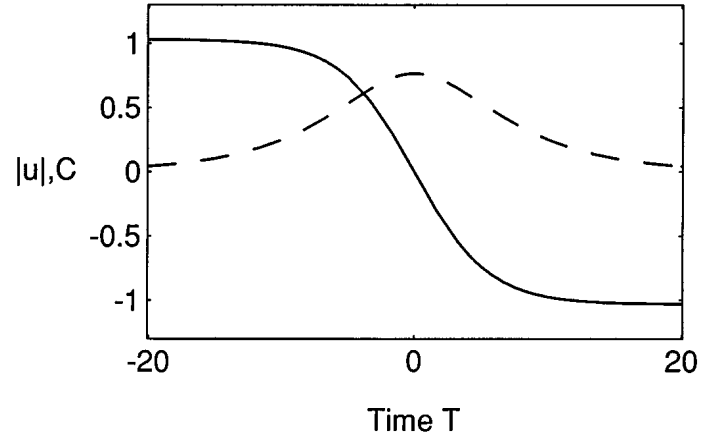

Fig. 9. Theoretical pulse amplitude $|u|$ (dashed curve) and its chirp $C$ (solid curve) versus time $T$ in the normal dispersion regime $(D=+1)$. 
ticular, it is quite clear that, whenever the excess gain $\delta>0$, the low-intensity background is unstable and therefore the solitary wave [Eqs. (3) and (4)] will eventually decay owing to its interaction with the exponentially amplified noise and radiation. On the other hand, it can be shown that this solution with $\delta<0$ is by itself unstable. ${ }^{16}$ Therefore for describing the pulsed operation of the laser it will be essential to introduce some stabilizing elements, such as higher-order nonlinear gain saturation terms and frequency sliding. We discuss the stability issue further in the following sections with the help of numerical simulations.

\section{NUMERICAL RESULTS}

We performed numerical simulations (by the split-step method) of pulse generation and propagation in an active fiber ring cavity of the type that was employed in the experiment (see Fig. 1). We assumed that nonlinear pulse propagation in the dispersive fiber span could be described by the lossy NLS equation

$$
\partial_{Z} U-\frac{i}{2} \partial_{T T} U-i|U|^{2} U=-\Gamma U,
$$

where $\Gamma=\gamma z_{0}$ is the dimensionless fiber loss coefficient [ $\gamma\left(\mathrm{m}^{-1}\right)$ is the fiber loss in real units]. We assumed for simplicity that the length of the active fiber, say, $z_{a} \ll z_{p}$, where $z_{p}$ is the length of the passive fiber span. Therefore we set the total dimensionless length of the laser loop equal to $Z_{l}=z_{l} / z_{0} \simeq z_{p} / z_{0}$. The fiber loss factor $\exp \left(-\Gamma Z_{p}\right)$ is overcompensated by the amplifier gain $\exp (G)$, i.e., the excess gain $\delta=G / Z_{p}-\Gamma>0$. We assumed that the spectral transfer function of the filter could be well approximated by the Fabry-Perot function $H\left(\Omega=\omega-\omega_{0}\right)$, where $\omega_{0}$ coincides with the center frequency of the filter and

$$
H(\Omega) \doteq \frac{1}{(1+2 i \Omega / B)},
$$

where $B$ is the filter bandwidth. The frequency shift that is obtained from the AOM may be simply represented by the transfer function in the time domain:

$$
S(T)=C \exp \left(i \alpha_{0} T\right),
$$

where $\alpha_{0}$ is the acoustic drive frequency. Note that the diffraction efficiency $I_{d} \doteq|C|^{2} \leq 1$ gives the fraction of energy that is transferred by the AOM into the frequencyshifted beam. By assuming that this extra loss which is due to the AOM is compensated by the active fiber, we may for simplicity set $C=1$. Finally, we have included in the simulations the presence of a fast saturable absorption mechanism (resulting, for example, from nonlinear polarization rotation plus polarization-dependent losses) by multiplying the field (after the amplifier) by the temporal transfer function

$$
G(T)=\exp \left[-\Gamma_{s} /\left(1+|U|^{2} / I_{s}\right)\right]
$$

For studying the stability of the soliton generation in the SFFL we took the initial condition for Eq. (5) of the form

$$
U(T, Z=0)=a_{0} \operatorname{sech}(T / A) .
$$

For example, we set $A=5$ and $a_{0}=1$. In order to fit the experimental results, in the simulations we chose the following parameters. The center wavelength of the filter was $\lambda_{0}=2 \pi c / \omega_{0}$, the loop length was $z_{l}=$ $70 \mathrm{~m}$, the fiber loss was $0.24 \mathrm{~dB} / \mathrm{km}$, the filter bandwidth was equal to $2.3 \mathrm{~nm}$ (or $290 \mathrm{GHz}$ ), the AOM frequency shift was $170 \mathrm{MHz}$, and the average loop dispersion was $\beta_{2}=+53 \mathrm{ps}^{2} / \mathrm{km}$. By taking the reference time width $t_{0}=1.4 \mathrm{ps}$, one obtains the dimensionless filter bandwidth $B=2.5$ in Eq. (6); the loop length $Z_{l}=z_{l} / z_{0}=2$, the average frequency shifting rate [see Eq. (2)] is $\alpha=$ $\alpha_{0} z_{0} / z_{l}=7.9 \times 10^{-4}$, whereas the average filter bandwidth in Eq. (2) is $\beta=0.167$ (note that $\beta$ is independent of the definition of $t_{0}$ ). With these parameters we found that an input condition of the form of Eq. (9) evolved toward a steady-state pulse with a full width at halfmaximum of $17 \mathrm{ps}$ (see Fig. 10). In the simulations we found that, given the reduced stabilizing effect of the small frequency shift rate $\alpha$, it was impossible to suppress the eventual growth of radiation waves with a positive average excess gain $\delta$. Therefore for the stability of the laser's steady state it was necessary to take $\delta<0$ and to include a fast saturable absorption mechanism of the type of Eq. (8). We set the gain coefficient of the active fiber $G=0.2$, whereas $\Gamma_{s}=0.22$ and $I_{s}=0.25$ in Eq. (8), so that the low-power average excess loss is $\delta=-0.01$. As can be seen from the contour plot of the pulse intensity of Fig. 11, the steady-state pulse that forms in the cavity is stable, and no growth of radiative waves is observed. Note from Figs. 10 and 11 that the pulse is slightly asymmetric, that is, the leading edge is steeper than the trailing edge. On the other hand, the asymmetric pulse spectrum in Fig. 10 shows that the high-frequency side of the nearly flat-topped spectrum falls down more sharply than the low-frequency side. We verified that this asymmetry does not change if the sign of the AOM frequency
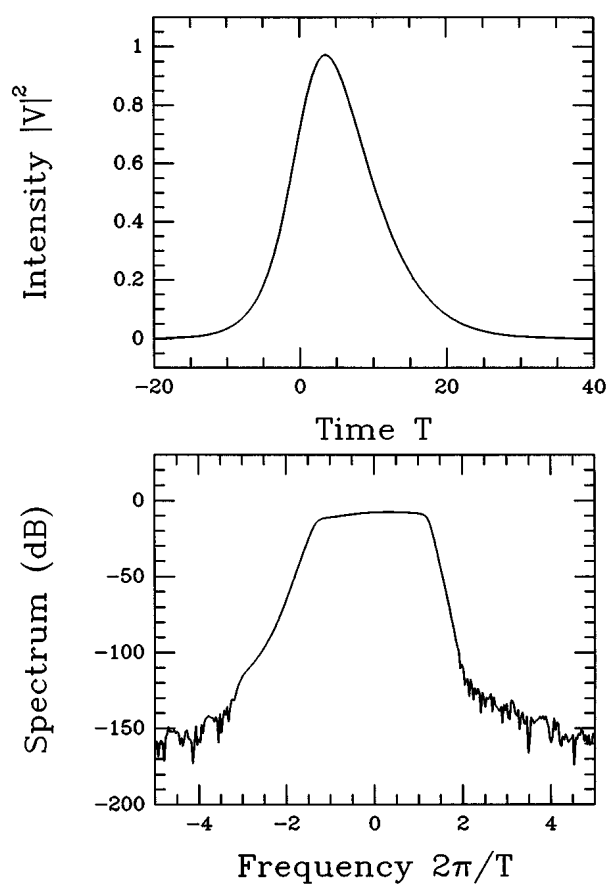

Fig. 10. Calculated intensity profile and spectrum of the steady-state pulses in the cavity in the normal dispersion regime and with fast saturable absorption. 


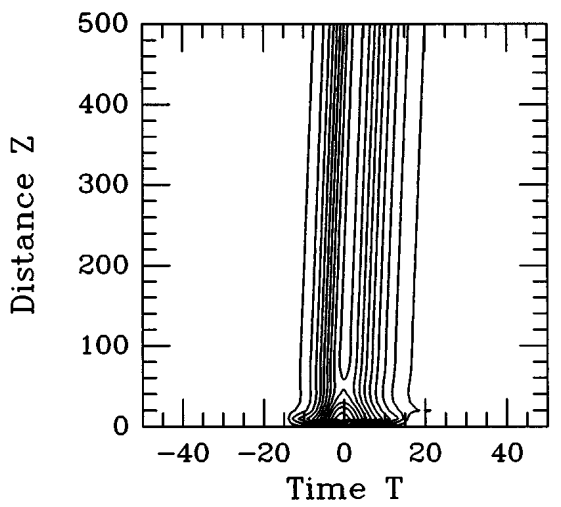

Fig. 11. Contour plot of the intensity of the steady-state pulse of Fig. 10.

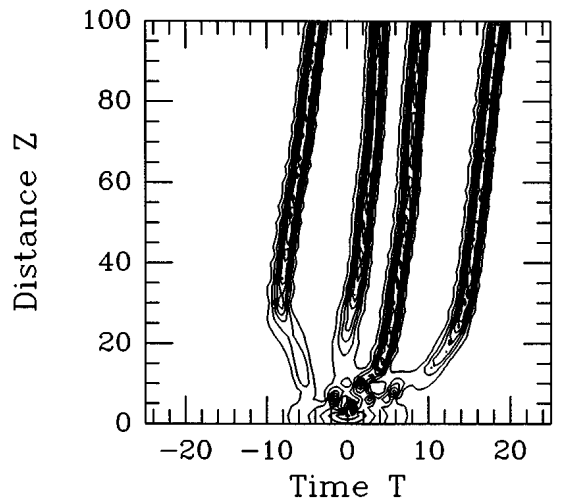

Fig. 12. Same as in Fig. 11 but with the sign of GVD reversed.

shift is inverted. Therefore the observed pulse asymmetry results from the third-order dispersion that is associated with the complex filter transfer function [Eq. (6)]. As we shall see in Section 4, the numerically predicted spectral asymmetry is well reproduced by the experimental observations. Note that the spectrum of the highly chirped pulse in Fig. 10 fills the entire bandwidth of the filter.

Finally, Fig. 12 shows the contour plot of the field intensity inside the laser, which is obtained for the same set of parameters as in the case of Fig. 11 but with an inverted sign of the average GVD, that is, $\beta_{2}=-53 \mathrm{ps}^{2} / \mathrm{km}$. As can be seen, in this case the initial pulse envelope [Eq. (9)] splits into a train of four equal pulses of 2-ps width. Therefore, by simply changing the sign of a relatively high GVD, one still obtains a stable pulse from the laser; however, the pulse width decreases approximately nine times.

Figure 13 shows the intensity profile and the spectrum of the stable steady-state pulses that are generated in the normal dispersion regime with a larger value of the averaged frequency-sliding rate $\alpha=-0.2$, the filter bandwidth $B=6, \delta=0.2$, and $Z_{l}=0.0864$. In this case, even though $\delta>0$, no saturable absorber was necessary because the stabilization of the cavity pulses was achieved through the relatively large rate of frequency upshifting from the AOM. In real units, the simulation that appears in Fig. 13 corresponds, for example, to the average cavity dispersion $\beta_{2}=+14 \mathrm{ps}^{2} / \mathrm{km}$ and the AOM frequency shift of $820 \mathrm{MHz}$, whereas the filter width and the cavity length are constant and equal to $2.3 \mathrm{~nm}$ and
$70 \mathrm{~m}$, respectively. Then the output pulse duration of Fig. 13 is equal to $20 \mathrm{ps.}$ One could also obtain the same situation by decreasing the filter bandwidth to $1 \mathrm{~nm}$, with $\beta_{2}=+74 \mathrm{ps}^{2} / \mathrm{km}$ and an AOM frequency shift of $356 \mathrm{MHz}$. In this case, the generated pulse duration would be equal to $45 \mathrm{ps}$. Note that the center of mass of the pulse spectrum in Fig. 13 is upshifted by the AOM with respect to the center frequency of the filter $\Omega=0$.

\section{EXPERIMENT}

In the SFFL the gain element was a 15-m-long erbiumdoped fiber that was pumped by a $70-\mathrm{mW} 980-\mathrm{nm}$ semiconductor laser (see Fig. 1). A polarization-insensitive isolator ensured the unidirectionality of the cavity, whereas we inserted polarization-dependent losses into the ring cavity by placing a polarization controller before a linear polarization. Continuous frequency shifting and filtering were achieved by means of a single integrated optics $\mathrm{LiNbO}_{3}$ component (see Fig. 2). The Mach-Zehnder geometry of the device leads to shifting and filtering of the light that is split (at point (2)in Fig. 2) in the orthogonal TE and TM polarization directions. The phase-matching condition of the coupling process between the acoustic and the light waves leads to frequency shifts with opposite signs for light that propagates in the two arms of the AOMF. In the laser loop we placed the polarizer before the AOMF, so that we fed light into a single arm of the device (see Fig. 1). The length of the AOMF was equal to half of the polarization beat length. This entails a 90-deg polarization rotation between the input and the output of the AOMF. The transmission bandwidth of the AOMF was $2.3 \mathrm{~nm}$, whereas the central transmission wavelength could be tuned in the range of 1525-1565 nm by adjustment of the acoustic wave frequency from 177 to $172.5 \mathrm{MHz}$. The total length of the fiber loop, with the exception of the dispersive nonlinear fiber span, was
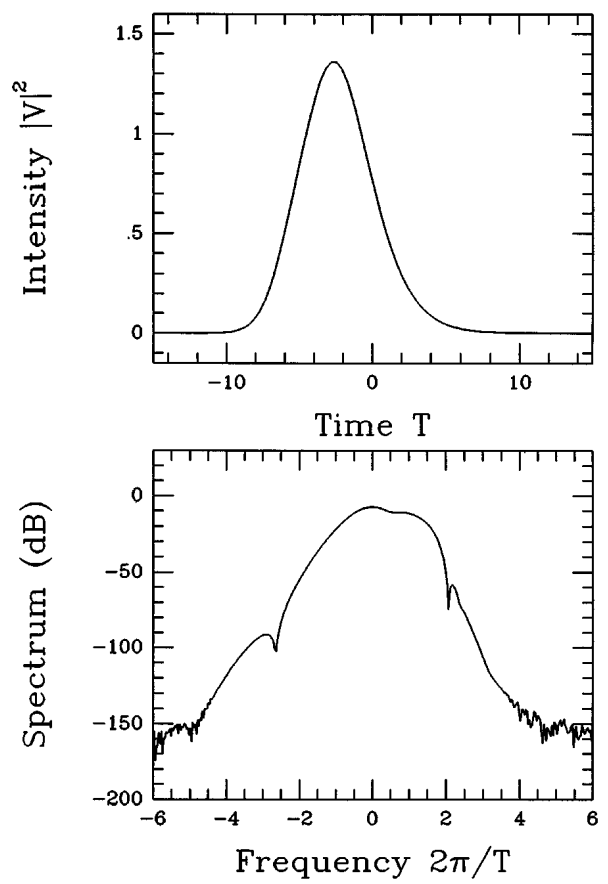

Fig. 13. Same as in Fig. 10 without fast saturable absorption and with a large frequency-shifting rate. 


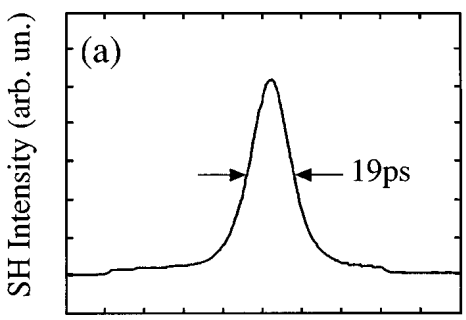

Delay $(500 \mu \mathrm{s} / \mathrm{div})$

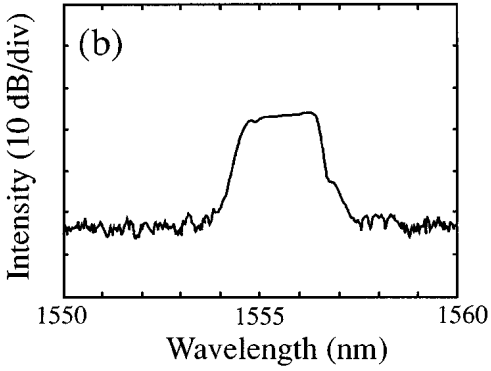

Fig. 14. (a) Experimental autocorrelation and (b) spectrum at the laser output. $\mathrm{SH}$, second harmonic.

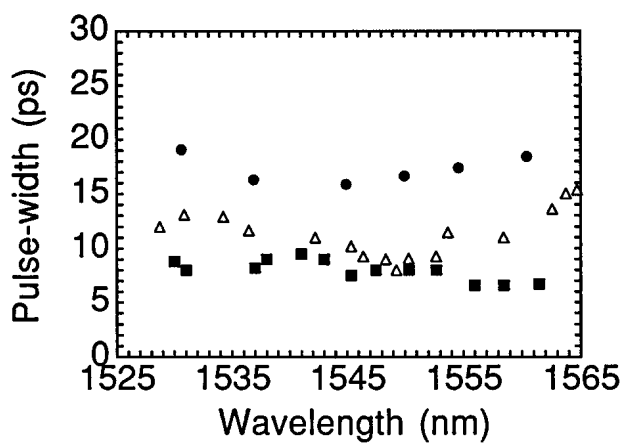

Fig. 15. Experimental dependence of laser pulse width versus operating wavelength for an average cavity GVD $\beta_{2}=-15.3 \mathrm{ps}^{2} / \mathrm{km}$ (squares), $\beta_{2}=+1 \mathrm{ps}^{2} / \mathrm{km}$ (triangles), and $\beta_{2}=+51.5 \mathrm{ps}^{2} / \mathrm{km}$ (circles).

$26.5 \mathrm{~m}$. The average chromatic dispersion of this part of the cavity was positive and was $\beta_{2}=+4 \mathrm{ps}^{2} / \mathrm{km}$.

In order to investigate the role of the average cavity dispersion in the characteristics of the emitted pulses, we repeated the experiment with three different nonlinear dispersive fiber spans. The first fiber, say, fiber A, was a standard 110-m-long fiber with the anomalous dispersion $\beta_{2}=-20 \mathrm{ps}^{2} / \mathrm{km}$. The second fiber (fiber B) was a 58-m-long dispersion-shifted fiber with $\beta_{2}=0$ at $1550 \mathrm{~nm}$. Finally, the third fiber (fiber C) was a $40-\mathrm{m}$ long special-purpose fiber with a large positive dispersion $\beta_{2}=+83 \mathrm{ps}^{2} / \mathrm{km}$.

Stable emission of picosecond pulses was observed in any case as soon as the pump power was turned on. In the normal dispersion regime the repetition rate of the generated pulses coincided with the travel time in the cavity. However, no adjustment between the acoustic drive frequency and the laser repetition rate was necessary for stable operation and self-starting of the laser, ${ }^{3,5}$ whereas we could easily change the mean frequency of the output pulses by tuning the central transmission wavelength of the AOMF. The investigation of the temporal and spectral characteristics of the output pulses was carried out by means of an autocorrelator and an optical spectrum analyzer. Figure 14 shows an example of the observed autocorrelation trace and spectrum in the case of a positive GVD cavity, that is, with fiber $\mathrm{C}$ in the loop. The measured time width of $19 \mathrm{ps}$ corresponds, when we assume for simplicity a symmetric hyperbolic secant profile pulse, to a real pulse full width of $12.4 \mathrm{ps}$. On the other hand, the width of the slightly asymmetrical flattopped spectrum coincides with the whole 2.3-nm filter bandwidth.

Figure 15 displays the dependence of the SFFL output pulse width on the mean wavelength with fiber A, $\mathrm{B}$, or $\mathrm{C}$ in the loop. With fiber $\mathrm{A}$ the average cavity GVD was $\beta_{2}=-15.3 \mathrm{ps}^{2} / \mathrm{km}$, with fiber B the average GVD was $+1 \mathrm{ps}^{2} / \mathrm{km}$ (at the zero-dispersion wavelength $\lambda=1550 \mathrm{~nm}$ ), and with fiber C the average GVD was $+51.5 \mathrm{ps}^{2} / \mathrm{km}$. The values of the pulse widths that correspond to the fibers $\mathrm{A}, \mathrm{B}$, and $\mathrm{C}$ are represented in Fig. 15 by squares, triangles, and dots, respectively. In each case, we adjusted the polarization controllers in the cavity to obtain the maximum reduction in the duration of the output pulses. As can be seen, the duration of the pulses from the laser did not show significant changes when the filter wavelength was tuned through the bandwidth of the active fiber. On the other hand, the output pulse width increased slightly when the cavity dispersion was varied from negative (squares) to zero (triangles), whereas it nearly doubled with the highly positive average cavity GVD (circles).

We made use of the temporal compression of the large and nearly linear pulse chirps of the steady-state SFFL pulses by means of an external fiber lead with anomalous GVD. First we boosted the peak power of the pulses from the laser by inserting an amplifier module providing $15 \mathrm{dBm}$ of saturated power after the output coupler. Subsequently we propagated the output pulses in a stan-
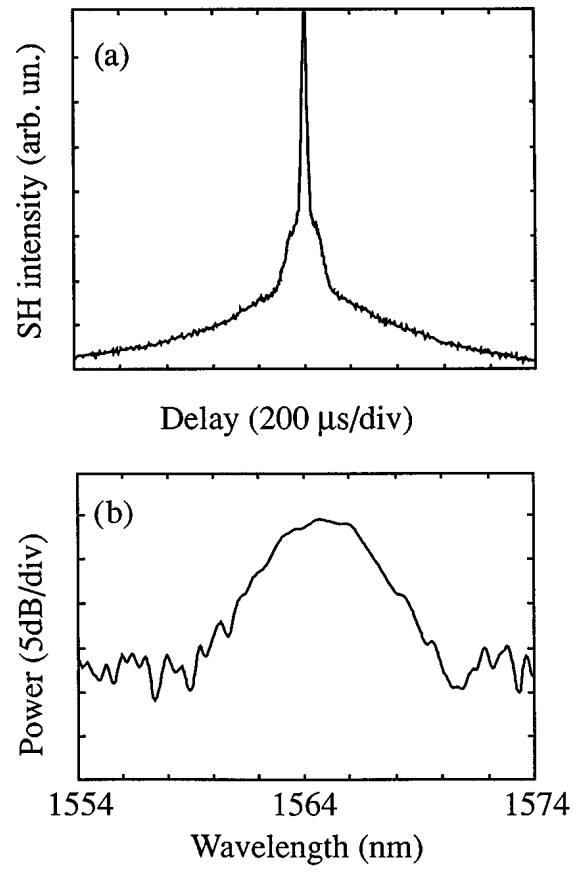

Fig. 16. (a) Autocorrelation and (b) spectrum of the compressed pulses from the fiber tail. SH, second harmonic. 
dard (of the type of fiber A that we mentioned above) anomalous dispersion fiber lead of length equal to $125 \mathrm{~m}$. Figure 16 shows the autocorrelation and the spectrum of the resulting time-compressed pulses. Their measured temporal width was $370 \mathrm{fs}$, which, when combined with its nearly Gaussian spectrum, leads to a nearly transformlimited time-bandwidth product of 0.41 .

\section{CONCLUSIONS}

In conclusion, in this research we have analyzed the role of group-velocity dispersion in the characteristics (duration, stability) of the pulses that are generated from a fiber ring laser with a filter and a continuous-frequency shift from an acousto-optical modulator. We have shown that the interplay of nonlinearity and bandwidth-limited amplification may lead to the formation of solitary pulses in both the normal and in the anomalous dispersion regimes. Fast saturable absorption as a result, for example, of nonlinear polarization rotation and polarizationdependent losses, may stabilize the pulse propagation process. Alternatively, frequency shifting may also stabilize the pulsed laser emission. We have obtained good agreement between the theory and the experimental observation of picosecond pulse generation in an erbiumdoped sliding-frequency fiber laser operating in the normal dispersion regime. We further exploited the uniform linear chirp across the cavity pulses for solitonic pulse compression of the laser output pulses down to less than 400-fs nearly transform-limited pulses.

\section{ACKNOWLEDGMENTS}

S. Wabnitz thanks E. Brinkmeyer for sending a preprint of his work ${ }^{4}$ before publication. The authors thank S. Bosso for having provided the acousto-optic shifter.
The research of $\mathrm{M}$. Romagnoli and $\mathrm{S}$. Wabnitz was carried out under the agreement between the Fondazione Ugo Bordoni and the Italian Post and Telecommunication Administration.

\section{REFERENCES}

1. P. D. Hale and F. V. Kowalski, IEEE J. Quantum Electron. 26, 1845 (1990).

2. H. Sabert and E. Brinkmeyer, in Optical Amplifiers and their Applications, Vol. 14 of 1993 OSA Technical Digest Series (Optical Society of America, Washington, D.C., 1993), paper TuD4; Electron. Lett. 29, 2122 (1993).

3. F. Fontana, L. Bossalini, P. Franco, M. Midrio, M. Romagnoli, and S. Wabnitz, Electron. Lett. 30, 321 (1994).

4. H. Sabert and E. Brinkmeyer, "Pulse generation in fiber lasers with frequency shifted feedback," J. Lightwave Technol. (to be published).

5. M. Romagnoli, S. Wabnitz, P. Franco, M. Midrio, F. Fontana, and G. Town, J. Opt. Soc. Am. B 12, 72 (1995).

6. Y. Kodama, M. Romagnoli, and S. Wabnitz, Electron. Lett. 30, 261 (1994)

7. L. F. Mollenauer, J. P. Gordon, and S. G. Evangelides, Opt. Lett. 17, 1575 (1992).

8. L. F. Mollenauer, E. Lichtman, M. J. Neubelt, and G. T. Harvey, Electron. Lett. 29, 910 (1993).

9. H. A. Haus, J. Appl. Phys. 46, 3049 (1975).

10. O. E. Martinez, R. L. Fork, and J. P. Gordon, J. Opt. Soc. Am. 2, 753 (1985).

11. P. A. Belanger, J. Opt. Soc. Am. B 8, 2077 (1991); H. A. Haus, J. G. Fujimoto, and E. P. Ippen, J. Opt. Soc. Am. B 8, 2068 (1991).

12. H. A. Haus, E. P. Ippen, and K. Tamura, IEEE J. Quantum Electron. 30, 200 (1994).

13. C.-J. Chen, P. K. A. Wai, and C. R. Menyuk, Opt. Lett. 19, 198 (1994)

14. N. R. Pereira and L. Stenflo, Phys. Fluids 20, 1733 (1977).

15. P. A. Belanger, L. Gagnon, and C. Paré, Opt. Lett. 17, 943 (1992).

16. C. Paré, L. Gagnon, and P. A. Belanger, Opt. Commun. 74, 228 (1989). 